we do not know. The search is already on for the geneticist's Rosetta Stone, that is, for a protein which can be analysed in the same detail as hæmoglobin, determined by a gene the fine structure of which can be resolved in the same detail as Benzer has resolved the rII locus in phage T4. But there are other problems to which no answer is at present in sight. One is the relationship between the structure of deoxyribonucleic acid, and the processes of chromosome replication and recombination; it is not clear at present whether replication and recombination are separate processes or different aspects of the same event. A still more fundamental difficulty is the relationship between gene action and morphogenesis. If the revolution in genetics achieves its present objectives, we shall know how a fertilized egg receives the instructions which tell it how to make a large number of specific proteins; but there is a big difference between a bag of proteins and an animal or plant.

J. MAYNARD SMITH

\section{A NEW APPROACH TO IMMUNITY}

The Clonal Selection Theory of Acquired Immunity By Sir Macfarlane Burnet. (The Abraham Flexner Lectures of Vanderbilt University, 1958.) Pp. ix +209 . (Cambridge: At the University Press; Nashville, Tennessee : Vanderbilt University Press, 1959.) 22s. $6 d$. net.

$\mathrm{T}$ HE basic problem of immunology is to understand how the body responds by making antibodies against foreign macromolecules, while refraining from making antibodies against the great variety of macromolecules which are present in its own tissues. No satisfactory instructive theory has been yet put forward to explain how the presence of the antigen causes cells to synthesize antibody molecules with a complementary surface structure. In the Abraham Flexner Lectures given at Vanderbilt University for 1958, and published in this book, Sir Macfarlane Burnet has approached the problem at quite a different angle. $H_{e}$ postulates that individual mesenchymal cells are genetically endowed with the potentiality for making globulin capable of combining with a particular antigenic configuration. In the adult organism, contact of the mesenchymal cell with the right antigen causes that cell to proliferate and to differentiate, so as to produce a clone of cells making, or capable of making, antibody which combines with the antigen. The mesenchymal cells are regarded as being subject to a high rate of somatic mutation, so that, when a clone proliferates, some members will produce globulin better adapted to the antigen, and these cells in turn will be stimulated selectively to proliferate, and so on. In this way, more and more cells will produce antibody capable of combining with more of, or more firmly with, the antigen surface. The problem of antibody production in response to an antigenic stimulus becomes therefore a problem in cell population dynamics, and the often amazing specificity of antibody for the antigen is achieved by a selective, rather than by an instructive, process. To account for the phenomenon of immunological toleranse, and the failure of antibodies to be formed against molecules present in the organism at birth, Burnet suggests that, during the stage of immunological immaturity, contact of antigen with cells potentially capable of making antibody against it results not in stimulation but in deletion of those clones. Hence the adult animal possesses cells capable only of being stimulated by molecules with surface configurations not shared by its own components. All other clones become forbidden.

If this ingenious theory is correct-in essence, even if not in detail - a fairly ready explanation is available not only for many of the cardinal features of immune responses, such as the difference between primary and secondary, or immunological memory and anamnestic reactions, but also for the development of auto-antibodies. According to the clonal solution hypothesis auto-antibodies could be evoked by body constituents which were screened from mesenchymal cells during the stage of immunological immaturity (for example, lens protein, or thyroglobulin), or which only developed later (for example, spermatozoa). They might also arise by somatio mutation in adult life of clones of cells capable of responding to forbidden patterns, such as to nucleic acid. Burnet makes the good point that antibodies are normally never formed against even heterologous deoxyribonucleic acid, but that when for some unknown reason they do appear, in persons with disseminated lupus erythematosus, they react with deoxyribonucleic acid from all sources including the nuclei of the sufferer's own cells. On the basis of his theory clones capable of making antibody against any deoxyribonucleic acid are forbidden, and eliminated, for the very reason that when antibody is formed, as a rare result of a somatic mutation, it will be an auto-antibody.

This example illustrates the self-consistency and the attractiveness of the theory. It is important to remember, however, that there is very little direct evidence to support its main assumption that clones of mesenchymal cells have a built-in response to a particular antigenic pattern, or to explain their deletion during embryonic life and stimulation later. The subsidiary hypothesis that lymphocytes, macrophages, plasma cells and primitive reticulum cells are all interconvertible is one the truth of which is convenient rather than proved, and so is the assumption that mesenchymal cells are hypermutable as regards somatic mutations. Biochemically minded readers may be disappointed by the frank rejection of a biochemical approach, and by the relegation to chance variations in a basic globulin structure of the problem of how antibody is synthesized with a pattern complementary to the antigen. Some may even be stimulated to try to show that a single cell can produce two or more unrelated antibodies at the same time-a finding which would be difficult to accommodate into the theory, and on which the evidence is at present conflicting.

The later chapters set out to show how the theory would apply to a wide range of phenomena from collagen disease to cancer, and to some of the latest experimental findings in immunology. Burnet manages with elegance in some cases, ingenuity in others, and occasionally a good deal of stretching to accommodate them all-including at least two experimental findings which have since become highly suspect or been withdrawn. This last is mentioned no more than as a warning that Sir Macfarlane has such enthusiasm and confidence in his latest ideas, that he can sweep up everything, including the readers, in his path. The ideas are, in any event, original and stimulating and have been carried further than anyone has tried to carry any of the alternative hypotheses. It is likely that a good deal of future work will be influenced by them, for their author has a reputation of having hit the right nail on the head on notable occasions in the past.
J. H. HUMPHREY 